\title{
Thermodynamic, Energy Efficiency, and Power Density Analysis of Reverse Electrodialysis Power Generation with Natural Salinity Gradients
}

\author{
Ngai Yin Yip, ${ }^{\dagger}$ David A. Vermaas, ${ }^{\ddagger}{ }^{\S}$ Kitty Nijmeijer, ${ }^{\S}$ and Menachem Elimelech ${ }^{* \dagger}$ \\ ${ }^{\dagger}$ Department of Chemical and Environmental Engineering, Yale University, New Haven, Connecticut 06520-8286, United States \\ ${ }^{*}$ Wetsus, Centre of Excellence for Sustainable Water Technology, P.O. Box 1113, 8900 CC Leeuwarden, The Netherlands \\ ${ }^{\S}$ Membrane Science \& Technology, University of Twente, MESA+ Institute for Nanotechnology, P.O. Box 217, 7500 AE Enschede, \\ The Netherlands
}

\section{Supporting Information}

ABSTRACT: Reverse electrodialysis (RED) can harness the Gibbs free energy of mixing when fresh river water flows into the sea for sustainable power generation. In this study, we carry out a thermodynamic and energy efficiency analysis of RED power generation, and assess the membrane power density. First, we present a reversible thermodynamic model for RED and verify that the theoretical maximum extractable work in a reversible RED process is identical to the Gibbs free energy of mixing. Work extraction in an irreversible process with maximized power density using a constant-resistance load is then examined to assess the energy conversion efficiency and power density. With equal volumes of seawater and river water,

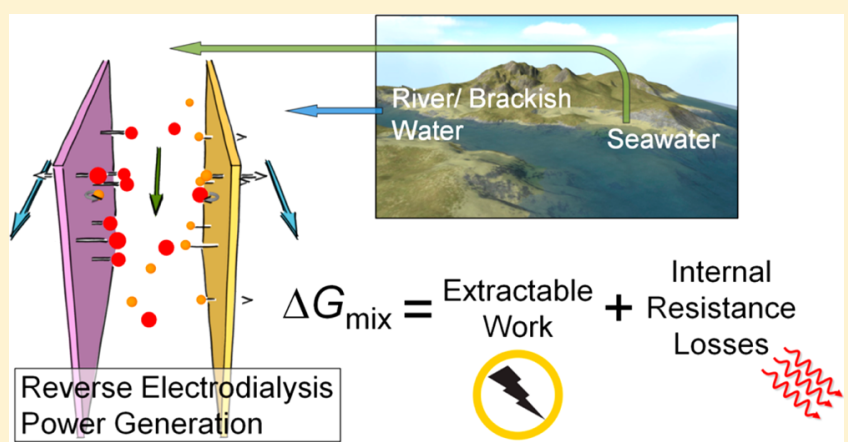
energy conversion efficiency of $\sim 33-44 \%$ can be obtained in RED, while the rest is lost through dissipation in the internal resistance of the ion-exchange membrane stack. We show that imperfections in the selectivity of typical ion exchange membranes (namely, co-ion transport, osmosis, and electro-osmosis) can detrimentally lower efficiency by up to $26 \%$, with co-ion leakage being the dominant effect. Further inspection of the power density profile during RED revealed inherent ineffectiveness toward the end of the process. By judicious early discontinuation of the controlled mixing process, the overall power density performance can be considerably enhanced by up to 7-fold, without significant compromise to the energy efficiency. Additionally, membrane resistance was found to be an important factor in determining the power densities attainable. Lastly, the performance of an RED stack was examined for different membrane conductivities and intermembrane distances simulating high performance membranes and stack design. By thoughtful selection of the operating parameters, an efficiency of $\sim 37 \%$ and an overall gross power density of $3.5 \mathrm{~W} / \mathrm{m}^{2}$ represent the maximum performance that can potentially be achieved in a seawater-river water RED system with low-resistance ion exchange membranes $\left(0.5 \Omega \mathrm{cm}^{2}\right)$ at very small spacing intervals $(50 \mu \mathrm{m})$.

\section{INTRODUCTION}

To meet current and future energy demands in a sustainable manner, alternative power sources that are clean and renewable need to be advanced. ${ }^{1}$ Nature's hydrological cycle offers a significant source of sustainable energy through salinity gradients. When two solutions of different concentrations are combined, the Gibbs free energy of mixing that is released can be harnessed for useful work. ${ }^{2,3}$ The annual global river discharge of $\sim 37300$ cubic $\mathrm{km}$ represents a currently untapped, selfreplenishing supply that can potentially be accessed for clean power generation. A recent study estimates that utilizing a tenth of the world's freshwater runoff can potentially generate electricity for over half a billion people with pressure retarded osmosis, a technology to convert the salinity energy to mechanical and then electrical energy. ${ }^{4}$

Reverse electrodialysis (RED) is another emerging technology that can harvest the energy from natural salinity gradients. 5,6
RED is the power generation analogue of electrodialysis (ED) separation. Whereas energy is consumed in ED to carry out separation of ions, ${ }^{7}$ RED utilizes the permeation of counterions across ion exchange membranes to generate a Nernst potential between two solutions of different concentration, while a redox couple at the end electrodes converts the ion flux into an electric current, thus producing energy through controlled mixing of the solutions. Essentially, RED operates as a "salinity battery", directly discharging stored chemical potential energy as electricity.

To advance the technology toward actual implementation, a better understanding of the potential RED process performance

Received: January 31, 2014

Revised: $\quad$ March 31, 2014

Accepted: April 3, 2014

Published: April 3, 2014 
at a system-level is necessary. Energy efficiency and membrane power density, performance indicators critical to the potential feasibility of RED energy production, had been evaluated in previous experimental studies. ${ }^{6,8-13}$ However, these ad hoc investigations are restricted to laboratory-scale batch experiments. Modeling efforts to simulate actual RED power generation have limited scope, focusing piecemeal on either efficiency $^{14-16}$ or power density ${ }^{16-19}$ optimization, with only one study concurrently examining both parameters. ${ }^{20}$ Furthermore, to date, there are no studies that quantify the individual impact of inevitable imperfections in membrane selectivity on energy efficiency and power density performance. Without a comprehensive grasp of the potential efficiency, and power density that can be simultaneously obtained, the energy capacity available from river runoffs mixing with the ocean and ionexchange membrane area needed to harness that energy cannot be accurately assessed. To realize the potential of RED power generation with natural salinity gradients, it is imperative that these gaps in our current knowledge of the process be addressed.

In this study, we carry out a robust analysis of a practical reverse electrodialysis process to systematically evaluate the energy efficiency and the concurrent power density. The theoretical maximum extractable work in RED is first determined from a reversible thermodynamic model and compared to the Gibbs free energy of mixing. We then evaluate the thermodynamic efficiency of work extraction and membrane power density in a practical RED process employing a constant-resistance external load. Membrane selectivity imperfections-co-ion transport, water osmosis, and electro-osmosis-are characterized, and their influence on the RED salinity battery performance is evaluated. Lastly, energy conversion efficiency and membrane power density are quantitatively assessed with simulated technologically available membranes. The intricate relationship between efficiency and power density is methodically examined and potential approaches to optimize the overall costeffectiveness of the technology are discussed. Our analytical study provides insights into the intrinsic efficiency and effectiveness of RED that can guide membrane module design and inform systems operation for the advancement of sustainable energy production from natural salinity gradients.

\section{REVERSIBLE THERMODYNAMIC MODEL OF REVERSE ELECTRODIALYSIS}

Reverse electrodialysis energy production with natural salinity gradients employs an RED stack, consisting of repeating pairs of cation-exchange membrane (CEM) and anion-exchange membrane (AEM), with high concentration (HC) and low concentration (LC) solutions alternatively flowing through each channel between the membranes (Figure 1A). ${ }^{5,6,11,12,21} \mathrm{~A}$ repeating cell consists of, in spatial order, a CEM, an HC solution compartment, an AEM, and an LC solution compartment (which is bordered by the CEM of the next adjacent cell). The salt (or ion) concentration difference across the ion-exchange membranes (IEMs) produces a Nernst potential. As the IEMs selectively allow the passage of counterions (i.e., cations for the negatively charged CEMs and anions for the positively charged AEMs), co-ions are retained while counterions permeate from the $\mathrm{HC}$ compartment to the LC compartment. Charge neutrality in the solutions is achieved due to the simultaneous permeation of cations and anions across the CEM and AEM, respectively, sandwiching each solution compartment. A pair of electrodes caps off the stack and a reversible redox couple (e.g., $\mathrm{Fe}^{2+} / \mathrm{Fe}^{3+}$ or $\left.\left[\mathrm{Fe}(\mathrm{CN})_{6}\right]^{4-} /\left[\mathrm{Fe}(\mathrm{CN})_{6}\right]^{3-}\right)^{22-24}$ is circulated between the
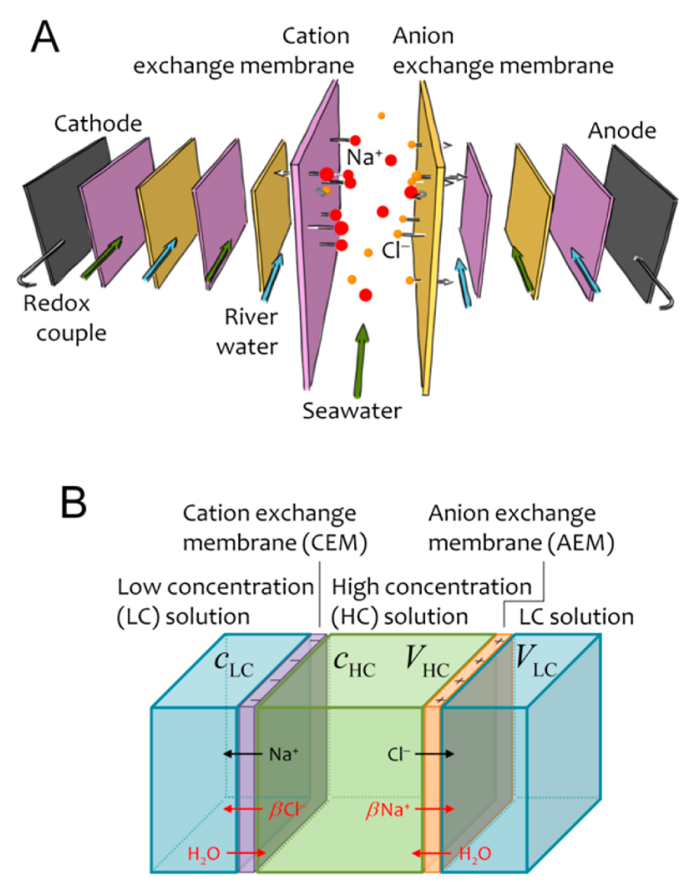

Figure 1. (A) Conceptual schematic of a reverse electrodialysis salinity battery. Four repeating stacks are shown, and a representative membrane pair is enlarged to illustrate the ion flux. The RED battery consists of repeating cells of alternating cation-exchange membrane (CEM) and anion-exchange membrane (AEM). High concentration (HC) and low concentration (LC) solutions, i.e., seawater and river or brackish water, respectively, flow through the channels alternatively. The ion-exchange membranes selectively allow the transport of counterions. The concentration difference across the ion-exchange membranes produces a Nernst potential. A redox couple circulating between the end electrodes converts the ion flux to an electric current with a reversible redox reaction. (B) Schematic of one RED cell, comprising a HC solution compartment (salt concentration $c_{\mathrm{HC}}$ and volume $V_{\mathrm{HC}}$ ) separated from two LC solution half-compartments (salt concentration $c_{\mathrm{LC}}$ and combined volume $V_{\mathrm{LC}}$ ) by a CEM possessing fixed negative charges and an AEM having fixed positive charges.

end electrodes to convert the ionic current to an electric current. The redox couple composition is maintained constant during the RED process as oxidation at the anode is exactly offset by reduction at the cathode.

In this section, we present a reversible thermodynamic model of RED and show the derivation of the theoretical maximum extractable work. In the following analyses, a $600 \mathrm{mM} \mathrm{NaCl} \mathrm{HC}$ solution is used to simulate seawater of $\sim 35 \mathrm{~g} / \mathrm{L}$ TDS, while the salt concentrations of the LC solutions are 1.5 and $17 \mathrm{mM} \mathrm{NaCl}$, to represent river water and brackish water of approximately 88 $\mathrm{mg} / \mathrm{L}$ and $1 \mathrm{~g} / \mathrm{L}$ TDS, respectively. ${ }^{4}$ The temperature is $298 \mathrm{~K}$ and assumed to remain constant throughout the RED process.

Reverse Electrodialysis Model. Figure 1B shows one RED cell, comprising an $\mathrm{HC}$ solution compartment separated from two LC solution half-compartments by a pair of CEM and AEM. The potential (or electromotive force, emf) of the cell, $\xi_{\text {emf }}$ is the sum of the Nernst potentials across the ion-exchange membrane pair due to the ion concentration difference

$$
\begin{aligned}
\xi_{\mathrm{emf}} & =\xi_{1 / 2 \mathrm{emf}, \mathrm{CEM}}+\xi_{1 / 2 \mathrm{emf}, \mathrm{AEM}} \\
& \approx\left(\frac{\alpha_{\mathrm{CEM}}}{z_{+}}+\frac{\alpha_{\mathrm{AEM}}}{z_{-}}\right) \frac{R_{\mathrm{g}} T}{F} \ln \frac{c_{\mathrm{HC}}}{c_{\mathrm{LC}}}
\end{aligned}
$$


where $\xi_{1 / 2 \mathrm{emf}}$ is the half-cell potential, $\alpha$ is the permselectivity of the IEM, $R_{\mathrm{g}}$ is the gas constant, $T$ is the absolute temperature, $z$ is the ion valence (e.g., $z=1$ for $\mathrm{Na}^{+}$and $\mathrm{Cl}^{-}$), and $F$ is the Faraday constant. The subscript of $\alpha$ and $\xi_{1 / 2 \mathrm{emf}}$ denotes cation- or anionexchange membrane, and the subscript of $z$ represents the cation $(+)$ or anion $(-)$, respectively. Details on the approximations to arrive at eq 1 are presented in the Supporting Information.

Although Figure 1B depicts a batch process, the model can also represent a continuous flow RED stack with the solutions circulated cocurrently at equal flow rates. Assuming ideal plugflow, the solution concentrations while advancing along the axial length of the RED membrane stack corresponds to the conditions in the batch process as controlled mixing progresses (i.e., the hydraulic residence time of the stack is equivalent to the mixing duration of the batch process).

For $\Delta n_{\mathrm{s}}$ moles of salt that has permeated (i.e., cations across the CEM and anions across the AEM), the molar salt concentration of the $\mathrm{HC}$ and LC solutions are $c_{\mathrm{HC}}=\left(n_{\mathrm{s}, \mathrm{HC}}^{0}-\right.$ $\left.\Delta n_{\mathrm{s}}\right) / V_{\mathrm{HC}}^{0}$ and $c_{\mathrm{LC}}=\left(n_{\mathrm{s}, \mathrm{LC}}^{0}-\Delta n_{\mathrm{s}}\right) / V_{\mathrm{LC}}^{0}$, respectively, where $n_{\mathrm{s}}$ is the moles of salt, $V$ is the solution volume, and superscript 0 denotes the initial solution. Note that the two LC halfcompartments in Figure $1 \mathrm{~B}$ are considered together as one solution volume, thus preserving electroneutrality. The IEMs are assumed to be water impermeable, and hence, the solution volume remains unchanged throughout the process (the effect of water osmosis is analyzed and discussed in a later section).

Substituting the expressions for $\mathcal{c}_{\mathrm{HC}}$ and $c_{\mathrm{LC}}$ into eq 1 , assuming $\alpha_{\mathrm{CEM}}=\alpha_{\mathrm{AEM}}=1$ (i.e., ion exchange membranes are perfectly selective for counterions), yields the electromotive force across the RED cell, $\xi_{\text {emf }}$, during the controlled mixing process. Figure $2 \mathrm{~A}$ shows a representative plot of $\xi_{\text {emf }}$ across the one-cell RED as a function of $\Delta n_{\mathrm{s}}$. Using equal volumes of seawater as the $\mathrm{HC}$ solution and river water as the LC solution (600 $\mathrm{mM}$ and $1.5 \mathrm{mM} \mathrm{NaCl}$, respectively), both $z_{+}$and $z_{-}$are unity and the initial potential difference across the CEM and AEM pair of the RED is calculated to be $308 \mathrm{mV}$ (vertical axis intercept). Actual seawater and river water contains some divalent ions (e.g., $\mathrm{Mg}^{2+}, \mathrm{Ca}^{2+}$, and $\mathrm{SO}_{4}{ }^{2-}$ ) that contribute less to the $\xi_{\text {emf }}$ compared to monovalent ions (as evident from the inspection of eq 1). For seawater and river water with $10 \%$ of salts as divalent ions, the $\xi_{\text {emf }}$ is $\sim 7 \%$ lower than the pure $\mathrm{NaCl}$ solutions used in this study. ${ }^{25}$

Ion transport proceeds until the $\mathrm{HC}$ and $\mathrm{LC}$ solutions are at equilibrium and the potential across the RED cell falls to zero (horizontal axis intercept in Figure 2A). The concentration of the final mixed solution, $c_{\mathrm{M}}^{f}$, can be determined by applying salt mass balance

$$
c_{\mathrm{M}}^{f}=\phi c_{\mathrm{LC}}^{0}+(1-\phi) c_{\mathrm{HC}}^{0}
$$

where $\phi$ is approximately the volumetric ratio of the initial LC solution to the total system volume: $\phi \approx V_{\mathrm{LC}}^{0} /\left(V_{\mathrm{LC}}^{0}+V_{\mathrm{HC}}^{0}\right){ }^{4}$ Details of the approximation are presented in the Supporting Information. The fraction of salt in the initial HC solution that is eventually transported across the IEMs can be calculated by solving $\Delta n_{\mathrm{s}}^{f}$ for $\mathcal{c}_{\mathrm{LC}}=c_{\mathrm{HC}}$

$$
\frac{\Delta n_{\mathrm{s}}^{f}}{n_{\mathrm{s}, \mathrm{HC}}^{0}}=\phi\left(1-\frac{c_{\mathrm{LC}}^{0}}{c_{\mathrm{HC}}^{0}}\right)
$$

where superscript $f$ denotes "final". Figure S1 (Supporting Information) shows representative plots of $\xi_{\text {emf }}$ across the onecell RED (eq 1) as a function of $\Delta n_{\mathrm{s}} / n_{\mathrm{s}, \mathrm{HC}}^{0}$ and $\Delta n_{\mathrm{s}} / \Delta n_{\mathrm{s}}^{f}$ for a

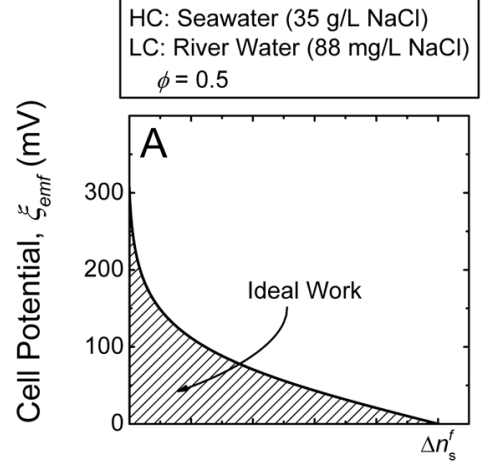

Moles of Salt Permeated, $\Delta n_{\mathrm{s}}$

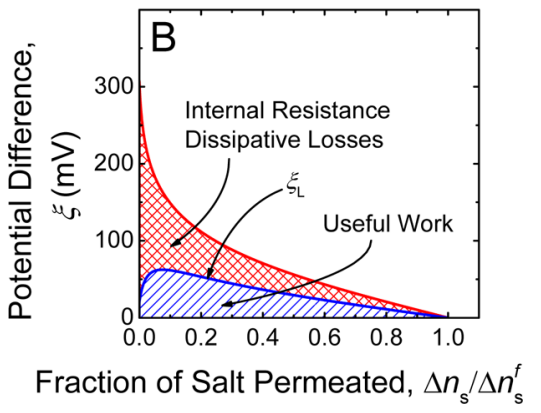

Figure 2. (A) Representative plot of the voltage (or electromotive force), $\xi_{\text {emff }}$ across the one-cell RED as a function of the moles of salt that permeated across the ion-exchange membranes, $\Delta n_{\mathrm{s}}$ (eq 1). In a reversible thermodynamic RED process, the potential difference across the external load is always exactly equal to $\xi_{\text {emf }}$. Multiplying the moles of salt permeated by the Faraday constant, $F$, and the total charge of the ions each salt molecule dissociates into (i.e., 2 for $\mathrm{NaCl}$ ) yields the charge transported, $\Delta q$. Hence, the total amount of work extractable from the hypothetical RED process, $W_{\text {ideal }}$, is equal to the area under the voltage-salt permeated (or, equivalently, $\xi-\Delta q$ ) plot (eq 5). (B) Representative plot of the useful work (blue patterned region), extractable in an RED process, where maximizing the overall membrane power density is the principal objective. The red patterned region marked "Internal Resistance Dissipative Losses" indicates the energy lost due to impedance caused by the RED stack resistance. In these representative plots, the $\mathrm{HC}$ solution is seawater $(600 \mathrm{mM}$ or $35 \mathrm{~g} / \mathrm{L}$ $\mathrm{NaCl})$, the $\mathrm{LC}$ solution is river water $(1.5 \mathrm{mM}$ or $88 \mathrm{mg} / \mathrm{L} \mathrm{NaCl})$, the volumetric fraction of the LC solution, $\phi$, is 0.5 , and temperature $T=$ $298 \mathrm{~K}$. The IEMs are assumed to be perfectly selective, the area specific resistance, ASR, of the membranes is $3.0 \Omega \mathrm{cm}^{2}$, and the intermembrane distance, $d$, is $150 \mu \mathrm{m}$.

range of $\phi$ values with the same seawater HC solution and river water LC solution.

Reversible Thermodynamic RED Process. In a theoretical reversible thermodynamic RED process, an infinitesimal ion flux is maintained throughout the controlled mixing. This is achieved by applying an opposite external potential that is negligibly smaller in magnitude to the cell emf. A very small amount of ions is transported across the membranes because of the infinitesimal resultant potential difference. The $\mathrm{HC}$ solution concentration is marginally lowered due to the salt permeation, while $c_{\mathrm{LC}}$ increases slightly. Hence, $\xi_{\text {emf }}$ minutely decreases such that it is now exactly equal to the external applied voltage.

The process of gradually lowering the external potential is repeated in infinitely small steps to achieve a continuous decrease while keeping the external potential virtually equal to $\xi_{\text {emf }}$. At any point during the reversible thermodynamic process, the magnitude of the external potential can be raised slightly above 
the cell emf, thus flipping around the ion flux direction and causing a small amount of ions to be transported back to the HC solution. Hence, the controlled mixing process is "reversed" and becomes a separation process (i.e., electrodialysis desalination). In actual operation, an RED process can approach reversible thermodynamics by connecting the stack to an external load of infinitely high resistance (relative to the stack) to achieve negligible ionic current.

Theoretical Maximum Extractable Work Is Equal to the Gibbs Energy of Mixing. In a hypothetical reversible thermodynamic RED process, no entropy is generated. ${ }^{26}$ The transfer of ionic charges, $\Delta q$, against the external applied voltage represents the theoretical maximum energy that can be extracted for useful work by the salinity battery. Integrating the external potential difference, which is precisely $\xi_{\text {emf }}$ for a reversible thermodynamic RED process, across the charges transported over the entire process yields the ideal work, $W_{\text {ideal }}$ :

$$
\begin{aligned}
W_{\text {ideal }} & =\int \xi_{\text {emf }} \mathrm{d} \Delta q \\
& =\nu_{+} z_{+} F \int_{0}^{\Delta n_{s}^{f}} \xi_{1 / 2 \mathrm{emf}, \mathrm{CEM}} d \Delta n_{\mathrm{s}}+\nu_{-} z_{-} F \int_{0}^{\Delta n_{s}^{f}} \xi_{1 / 2 \mathrm{emf}, \mathrm{AEM}} \mathrm{d} \Delta n_{\mathrm{s}}
\end{aligned}
$$

To account for cation transport across the CEM potential and anion flux across the AEM potential, the integral is split into the contribution from each membrane. The moles of salt permeated, $\Delta n_{\mathrm{s}}$, can substitute for the variable of integration $\left(\Delta q_{ \pm}=\right.$ $\left.\nu_{ \pm} z_{ \pm} F \Delta n_{\mathrm{s}}\right)$.

Substituting eq 1 and the expressions for $c_{\mathrm{HC}}$ and $c_{\mathrm{LC}}$ into eq 4 gives the ideal work extractable in RED

$$
\begin{aligned}
W_{\text {ideal }} & =\nu R_{\mathrm{g}} T \int_{0}^{\Delta n_{\mathrm{s}}^{f}} \ln \frac{\mathcal{c}_{\mathrm{HC}}}{c_{\mathrm{LC}}} \mathrm{d} \Delta n_{\mathrm{s}} \\
& =\nu R_{\mathrm{g}} T \int_{0}^{\Delta n_{\mathrm{s}}^{f}}\left(\ln \frac{n_{s, \mathrm{HC}}-\Delta n_{s}}{V_{\mathrm{HC}}^{0}}-\ln \frac{n_{s, \mathrm{LC}}+\Delta n_{s}}{V_{\mathrm{LC}}^{0}}\right) \mathrm{d} \Delta n_{\mathrm{s}}
\end{aligned}
$$

where $\nu=\nu_{+}+\nu_{-}$is the number of ions each salt molecule dissociates into (e.g., $\nu$ is 2 for $\mathrm{NaCl}$ ). Here, the IEMs were taken to be perfectly selective (i.e., $\alpha_{\mathrm{CEM} / \mathrm{AEM}}=1$ ); the influence of coion leakage is examined in a later section. A graphical representation of $W_{\text {ideal }}$ is given by area under the voltage $-\Delta n_{\mathrm{s}}$ plot as depicted in Figure 2A. The integral can be solved using eq 3 for the limits of the integration to arrive at the specific ideal work, $W_{\text {ideal, }, V_{\mathrm{LC}}^{0}}$ defined as energy per unit volume of the initial LC solution:

$$
\begin{aligned}
-\frac{W_{\text {ideal }, V_{\mathrm{LC}}^{0}}}{\nu R_{\mathrm{g}} T} & =\frac{c_{\mathrm{M}}^{f}}{\phi} \ln c_{\mathrm{M}}^{f}-c_{\mathrm{LC}}^{0} \ln c_{\mathrm{LC}}^{0}-\frac{(1-\phi)}{\phi} c_{\mathrm{HC}}^{0} \ln c_{\mathrm{HC}}^{0} \\
& =-\frac{\Delta G_{\text {mix }, V_{\mathrm{LC}}^{0}}}{\nu R_{\mathrm{g}} T}
\end{aligned}
$$

The negative sign of $W_{\text {ideal, }, V_{\mathrm{LC}}^{0}}$ signifies that work is being done by the one-cell RED system.

The expression for $W_{\text {ideal, }, V_{\mathrm{LC}}^{0}}$ in eq 6 is identical to the Gibbs free energy of mixing per unit volume of the LC solution, $\Delta G_{\mathrm{mix}, V_{\mathrm{LC}}^{0}}{ }^{4}$ This result is expected and is consistent with the principles of thermodynamics - the change in the Gibbs free energy of a system is equivalent to the work done by the system in an ideal reversible thermodynamic process. ${ }^{26,27}$ The specific ideal work, or the free energy of mixing per unit initial volume of the more dilute solution, as a function of the volumetric fraction of the LC solution, $\phi$, is shown in Figure S2 of the Supporting
Information for seawater as the salty stream and river water and brackish water as the fresh stream.

\section{ENERGY EFFICIENCY OF REVERSE ELECTRODIALYSIS}

A hypothetical reversible thermodynamic RED process would require infinite membrane area or unlimited time because of the infinitesimal rate of controlled mixing and is, hence, impractical to implement. Actual processes are not completely reversible due to the inevitable production of entropy. ${ }^{26}$ Entropy production, as dictated by the second law of thermodynamics, subtracts from $W_{\text {ideal }}$ and, thus, reduces the amount of energy accessible for conversion to useful work. In this section, we introduce operational considerations in a practical process to the RED model and analyze the power generation efficiency with natural salinity gradients.

Internal Resistances in RED Power Generation Circuit. In an actual RED salinity battery, the stack components are not ideally conductive but possess resistance that impedes the ionic and electric current. The resistance of the stack depicted in Figure $1 \mathrm{~A}, r_{\text {stack }}$ is the sum of the stack elements in series. $5,6,8,28$ For the one repeating RED cell considered in Figure 1B, the area specific resistance, $\mathrm{ASR}_{\text {cell }}$, is the product of $r_{\text {stack }}$ and the effective cell area, $A$, divided by the number of membranes pairs (or repeating RED cells), $N$,

$$
\begin{aligned}
\mathrm{ASR}_{\text {cell }} & =r_{\text {cell }} A=\frac{r_{\text {stack }} A}{N} \\
& \approx \mathrm{ASR}_{\mathrm{AEM}}+\mathrm{ASR}_{\mathrm{CEM}}+\frac{1}{\Lambda}\left(\frac{d_{\mathrm{HC}}}{c_{\mathrm{HC}}}+\frac{d_{\mathrm{LC}}}{c_{\mathrm{LC}}}\right)
\end{aligned}
$$

where $\Lambda$ is the molar conductivity of electrolyte $(\mathrm{NaCl})$ solution, $d$ is the intermembrane distance, and $c$ is the solution molar concentration. The product of molar conductivity and concentration yields the solution conductivity $(\kappa=\Lambda c)$. For the $\mathrm{NaCl}$ salt concentrations considered in this study, $\Lambda$ is calculated to be $0.08798 \mathrm{mS} \mathrm{cm} \mathrm{cm}^{-1} \mathrm{mM}^{-1}$ from the linear regression of $\kappa$ against $c\left(R^{2}=0.996\right.$ for $\left.0<c<1 \mathrm{M} \mathrm{NaCl}\right) .{ }^{29}$ The resistive property of the IEMs is described by the area specific resistance, ASR; dividing ASR by $A$ yields the membrane resistance (i.e., larger membrane areas contribute less resistance to the stack).

The four terms on the right denote the contribution to resistance from AEM, CEM, HC solution compartment, and LC solution compartment, respectively, as indicated by the subscripts. For RED salinity batteries with a large number of repeating membrane pairs, the contribution of the resistance of the end electrodes and redox couple compartment to one RED cell is relatively small due to normalization by $N$ and, thus, can be neglected. ${ }^{8}$ The resistance of the elements is assumed to be ohmic. $^{5,20}$ A past study reports that membrane resistance is detrimentally elevated in very dilute solutions $(<50 \mathrm{mM}$ $\mathrm{NaCl}) .{ }^{28}$ However, the final mixed solution concentration (eq 2 ) of almost the entire range of scenarios examined here $(0<\phi<$ $0.92)$ is greater than $50 \mathrm{mM}$. Thus, the analysis is simplified by taking membrane resistance, $\mathrm{ASR}_{\mathrm{AEM}}$ and $\mathrm{ASR}_{\mathrm{CEM}}$ of eq 7, to be independent of the surrounding salt concentration. Additionally, concentration polarization at the boundary layer of the membrane-solution interface can detrimentally add resistance to the cell. Here, we assume adequate mixing such that the effects of concentration polarization are negligible. ${ }^{14,18,30}$ The potential blocking of effective membrane area by nonconductive spacers, i.e., spacer shadow effect, is not considered. Practically, spacer 
shadow effects can be suppressed by using profiled membranes or very open spacers. ${ }^{30}$

During RED, ions permeate across the membranes and $c_{\mathrm{HC}}$ declines while the LC solution salt concentration rises. The last two terms of eq 7 change, and therefore, the area specific resistance of the cell varies as RED progresses. Figure S3 of the Supporting Information shows representative plots of $\mathrm{ASR}_{\text {cell }}$ as a function of the fraction of salt permeated, $\Delta n_{\mathrm{s}} / \Delta n_{\mathrm{s}}^{f}$. The ASR of the CEMs and AEMs is taken to be $3.0 \Omega \mathrm{cm}^{2}$ (typical values of commercial and laboratory-fabricated IEMs reported in literature ranges between $\left.\sim 0.7-11 \Omega \mathrm{cm}^{2}\right),{ }^{31,32}$ while the distance between the AEM and CEM, $d$, is $150 \mu \mathrm{m}$ (intermembrane distance of $\sim 60-500 \mu \mathrm{m}$ has been investigated in previous RED studies). $6,8,13,20$

Discussion of the RED cell resistance over the duration of the controlled mixing process is detailed in the Supporting Information. Briefly, $\mathrm{ASR}_{\text {cell }}$ is highest at the beginning of the process due to the low initial salt concentration of the LC solution (Figure S3, Supporting Information). As ions are transported into the LC solution, the compartment becomes more conductive and the overall impedance decreases correspondingly. Because the resistance of the IEMs is assumed to be constant during $\mathrm{RED}, \mathrm{ASR}_{\mathrm{AEM}}+\mathrm{ASR}_{\mathrm{CEM}}$ gradually begin to dominate $\mathrm{ASR}_{\text {cell }}$ beyond a certain point when $d_{\mathrm{LC}} / \Lambda c_{\mathrm{LC}}$ becomes relatively small. Therefore, membrane conductivity is anticipated to play an important role in RED performance.

Work Extraction with Constant-Resistance External Load. A simple electric circuit is employed to analyze the energy efficiency, where an external load of constant ohmic resistance, $R_{\mathrm{L}}$, is connected to the RED salinity battery to perform useful work; ${ }^{5,21}$ the external resistor is in series with the internal cell resistance, $r_{\text {cell }}$ (Figure S4, Supporting Information). The ionic current in the RED cell is assumed to be completely converted to an electric current, $I$, without any losses.

Based on Ohm's law, the governing equation for current density (or equivalently, the molar ion flux multiplied by the ion valence and the Faraday constant) across the RED cell, $i$, is

$$
i \equiv \frac{I}{A}=\frac{\xi_{\mathrm{emf}}}{A R_{\mathrm{L}}+\mathrm{ASR}_{\text {cell }}}=\frac{\xi_{\mathrm{emf}}-\xi_{\mathrm{L}}}{\mathrm{ASR}_{\text {cell }}}
$$

where $\xi_{\mathrm{L}}=R_{\mathrm{L}} \xi_{\text {emf }} /\left(R_{\mathrm{L}}+r_{\text {cell }}\right)$ is the potential difference across the load. It is worthwhile to note that eq 8 takes the general form of the water flux equation in pressure retarded osmosis power generation. $^{33,34}$ Specifically, parameters $i, \mathrm{ASR}_{\text {cell }}{ }^{-1}, \xi_{\text {emf }}$, and $\xi_{\mathrm{L}}$ are analogous to water flux, membrane water permeability, osmotic pressure difference across the PRO membrane, and the applied hydraulic pressure, respectively. As salt permeates across the membranes, the cell potential decreases according to eq 1 (as illustrated in Figure 2A), and changes in $c_{\mathrm{HC}}$ and $c_{\mathrm{LC}}$ also cause an overall reduction in $r_{\text {cell }}$ (eq 7, Figure S3, Supporting Information). Hence, when $R_{\mathrm{L}}$ is fixed, $\xi_{\mathrm{L}}$ varies nonlinearly as RED proceeds.

The extractable work in actual RED, $W$, is the integral of $\xi_{\mathrm{L}}$ across the charges transported:

$$
W=\int \xi_{\mathrm{L}} \mathrm{d} q=\nu R_{g} T \int_{0}^{\Delta n_{s}^{f}} \frac{R_{\mathrm{L}} A}{R_{\mathrm{L}} A+\mathrm{ASR}_{\text {cell }}} \ln \frac{c_{\mathrm{HC}}}{c_{\mathrm{LC}}} \mathrm{d} \Delta n_{s}
$$

Similar to the earlier integration (eq 4), the moles of salt permeated across the IEMs, $\Delta n_{s}$, can substitute for $\Delta q$. Inspection of eqs 5 and 9 reveals that the actual work is less than $W_{\text {ideal }}$ as $R_{\mathrm{L}} A /\left(R_{\mathrm{L}} A+\mathrm{ASR}_{\text {cell }}\right)<1$. By using an external load of extremely large resistance, the fraction tends to unity and $W$ approaches the ideal work (i.e., current is suppressed to virtually zero and the process approaches reversible thermodynamics).

Maximum Power Density in Practical RED Operation. Membrane power density, PD, defined as the power generated per unit total membrane area (i.e., both CEMs and AEMs), is a key factor in determining the economical feasibility of RED energy production with natural salinity gradients. ${ }^{8,10,14,35}$ For the one-cell RED model presented in this analysis, the instantaneous power density, $\mathrm{PD}$, is

$$
\mathrm{PD}=\frac{P_{\mathrm{L}}}{2 A}=\frac{1}{2}\left(\frac{\xi_{\mathrm{emf}}}{R_{\mathrm{L}} A+\mathrm{ASR}_{\text {cell }}}\right)^{2}\left(R_{\mathrm{L}} A\right)
$$

where $P_{\mathrm{L}}$ is the useful power generated by the external load and the factor of $1 / 2$ accounts for the ion exchange membrane pair. As $P_{\mathrm{L}}=I^{2} R_{\mathrm{L}}$ (where $I$ is the electric current) and $\mathrm{ASR}_{\text {cell }}=r_{\text {cell }} A$, the power density at a particular moment can be expressed as a function of the RED cell potential, $\xi_{\text {emf }}$ the area specific resistance of the cell, and the product of the load resistance and cell area, which is analogous to ASR. Again, as both $\xi_{\text {emf }}$ and $\mathrm{ASR}_{\text {cell }}$ change during RED, the instantaneous power density has a nonlinear dependence on $\Delta n_{\mathrm{s}}$.

The maximum PD (or, equivalently, $P_{\mathrm{L}}$ ) is obtained when the external load resistance is equal to the internal resistance. However, this would necessitate $R_{\mathrm{L}}$ to vary continuously in order the match the changing $\mathrm{ASR}_{\text {cell }}$ (eq 7) as RED proceeds. The equivalent implementation in an actual process would require the RED stack to be divided into infinite segments. An external load having resistance specifically tailored to the local solution concentrations is connected to each segment so as to attain $R_{\mathrm{L}}$ $=\mathrm{ASR}_{\text {cell }} / A$ at all points along the channel. ${ }^{20}$ Such a configuration is unfeasibly complicated and, hence, a more practical approach is employed for this analysis using a single external load of constant ohmic resistance.

The net useful work divided by the entire duration of the RED process gives the average power of the load, and further normalization by the total membrane area, $2 \mathrm{~A}$, yields the overall membrane power density, $\mathrm{PD}_{\text {avg }}$

$$
\mathrm{PD}_{\mathrm{avg}}=\frac{\int_{0}^{\Delta q^{f}} \xi_{\mathrm{L}} \mathrm{d} \Delta q}{2 A \int_{0}^{\Delta q^{f}} R_{\mathrm{L}} / \xi_{\mathrm{L}} \mathrm{d} \Delta q}
$$

where $\int \xi_{\mathrm{L}} \mathrm{d} \Delta q$ is work, $W$ (eq 9). The current $(\mathrm{d} \Delta q / \mathrm{d} t)$ during the process is $\xi_{\mathrm{L}} / R_{\mathrm{L}}$. Thus, the integral of $R_{\mathrm{L}} / \xi_{\mathrm{L}}$ (inverse of current) across the charges permeated in the denominator is $\int(\mathrm{d} t / \mathrm{d} \Delta q) \mathrm{d} \Delta q$, the duration of the controlled mixing process. An inspection of eqs 9 and 11 reveals that a small load resistance will expedite the controlled mixing but produces less useful work, whereas a large $R_{\mathrm{L}}$ generates a greater $W$ but at the expense of a longer duration (or equivalently, the residence time in a RED stack). Hence, the optimum load resistance, $R_{\mathrm{L}}^{*}$, that maximizes the overall power density is obtained by solving for $d \mathrm{PD}_{\text {avg }} / d R_{\mathrm{L}}$ equals to zero. The calculated $R_{\mathrm{L}}^{*}$ is then substituted into eq 9 to find the specific work when power density is maximized, $W^{*}$. All integrals and derivatives were analyzed numerically. In theory, RED requires infinite time for absolute completion because the rate of ion transport approaches zero as the process nears the end (i.e., $\xi_{\mathrm{L}}$ tends to zero and $R_{\mathrm{L}} / \xi_{\mathrm{L}} \rightarrow \infty$ ). To circumvent obtaining a trivial solution for $\mathrm{PD}_{\text {avg }}$ the process was terminated after 99.99\% of $\Delta n_{\mathrm{s}}^{f}$ was transferred. 


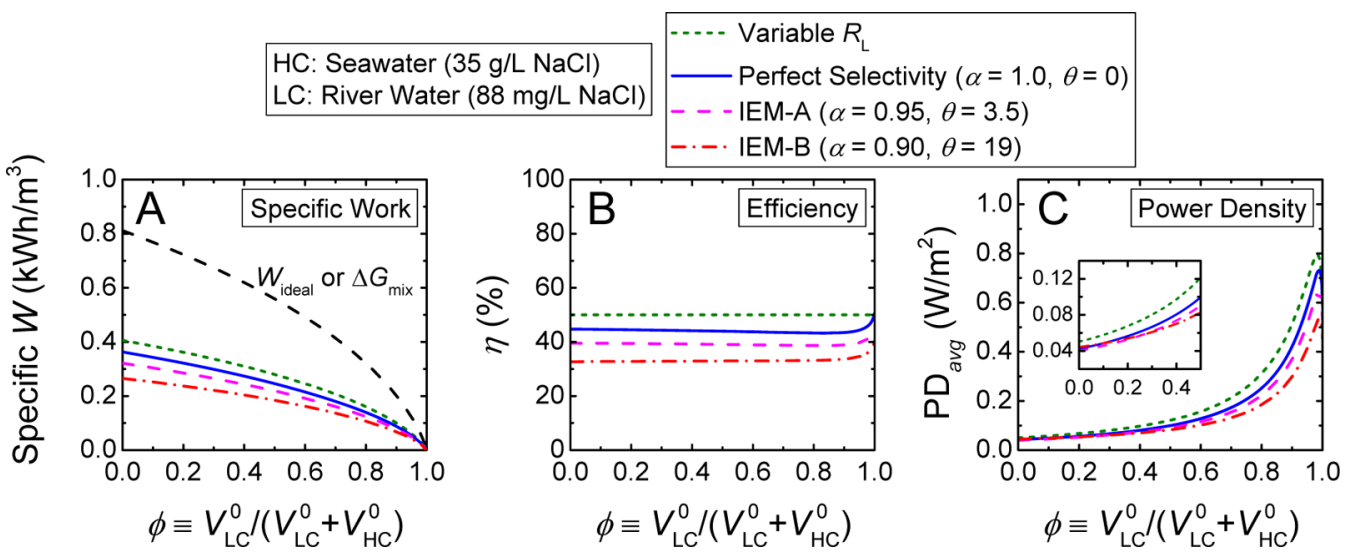

Figure 3. (A) Extractable work per unit volume of the LC solution, specific $W^{*}$, (B) energy conversion efficiency, $\eta$, and (C) overall membrane power density of RED energy production, $\mathrm{PD}_{\text {avg }}$, as a function of the volumetric fraction of $\mathrm{LC}$ solution to both $\mathrm{LC}$ and $\mathrm{HC}$ solutions, $\phi$. The dashed black line in (A) indicates the specific ideal work extractable in a reversible thermodynamic RED process, $W_{\text {ideal, } V_{\mathrm{LC}}^{0}}$, which is equivalent to the specific Gibbs free energy of mixing, $\Delta G_{\text {mix }, V_{L C}{ }^{\circ}}$. The solid blue lines, dashed magenta lines, and dash-dotted red lines indicate a perfectly selective membrane (i.e., $\alpha=1$ and $\theta$ $=0)$, IEM-A with moderately imperfect selectivity $(\alpha=0.95$ and $\theta=3.5)$, and IEM-B with more severe selectivity imperfections $(\alpha=0.90$ and $\theta=19)$, respectively. The dotted green lines indicate the theoretical performance achieved with perfectly selective membranes when the resistance of the external load is varied such that it always exactly matches the changing RED stack resistance (i.e., $R_{\mathrm{L}}=r_{\text {cell }}$ ). In this hypothetical case, $\eta$ is always $50 \%$ and PD is maximized. A magnified plot of $(\mathrm{C})$ for $0<\phi<0.5$ is shown in the inset. Seawater $(600 \mathrm{mM}$ or $35 \mathrm{~g} / \mathrm{L} \mathrm{NaCl})$ is employed as the $\mathrm{HC}$ solution, river water $(1.5 \mathrm{mM}$ or $88 \mathrm{mg} / \mathrm{L} \mathrm{NaCl})$ is used as the LC solution, and temperature $T=298 \mathrm{~K}$. The area specific resistance, ASR, of the membranes is $3.0 \Omega \mathrm{cm}^{2}$ and the intermembrane distance, $d$, is $150 \mu \mathrm{m}$.

Energy Conversion Efficiency. The potential difference across an external load as a function of $\Delta n_{\mathrm{s}} / \Delta n_{\mathrm{s}}^{f}$, for a seawaterriver water system is shown in Figure $2 \mathrm{~B}$ (solid blue line). The optimal load resistance $R_{\mathrm{L}}^{*} A$ is $8.29 \Omega \mathrm{cm}^{2}$ and $\phi=0.5$. The work extractable with RED is represented by the area under the $\xi_{\mathrm{L}}$ curve and is obtained from eq 9. The energy conversion efficiency, defined as the percentage of the Gibbs free energy of mixing (or ideal work) that can be extracted in actual RED when power density is maximized $\left(\eta=W^{*} / \Delta G_{\text {mix }} \times 100 \%\right)$, can be determined using eqs 6 and 9. In this investigation, only membrane-level phenomena are considered in the determination of efficiency, while system-level inefficiencies (such as parasitic hydraulic pressure drop and overpotential at the end electrodes), ${ }^{18,22}$ which will further lower the overall energy conversation performance, are not incorporated.

Figure 3 shows the specific work (extractable energy per unit $V_{\mathrm{LC}}^{0}$ ), energy efficiency, $\eta$, and overall membrane power density, $\mathrm{PD}_{\mathrm{avg}}$, as a function of the volumetric fraction of LC solution to both LC and HC solutions, $\phi$. Model seawater $(600 \mathrm{mM}$ or $35 \mathrm{~g} /$ $\mathrm{L} \mathrm{NaCl}$ ) was employed as the $\mathrm{HC}$ solution, and $1.5 \mathrm{mM}$ or 88 $\mathrm{mg} / \mathrm{L} \mathrm{NaCl}$ was employed as the $\mathrm{LC}$ solution to simulate river water. The analysis assumes perfectly selective ion-exchange membranes (solid blue lines) that completely exclude co-ions and water. Imperfections in membrane selectivity are discussed in a later section. The specific ideal work (or, equivalently, the Gibbs free energy of mixing) is indicated in Figure 3A as the dashed black line for comparison.

For the seawater-river water RED system with perfectly selective membranes, the amount of energy extractable per unit volume of the river water is highest at small $\phi$ values (specific $W^{*}$ $=0.36 \mathrm{kWh} / \mathrm{m}^{3}$ and $\eta=44.8 \%$ when $\phi$ approaches zero) and $W^{*}$ diminishes to zero as $\phi$ increases to unity (Figure $3 \mathrm{~A}$ ). The dotted green lines indicate $W, \eta$, and $\mathrm{PD}_{\text {avg }}$ when the external load resistance varies throughout the controlled mixing process such that it exactly matches the internal resistance to achieve the greatest possible overall power density. The resulting 50\% efficiency of this hypothetical scenario (regardless of $\phi$ ) represents the idealized $\eta$ that can be achieved when $\mathrm{PD}_{\text {avg }}$ is maximized (Figure 3B).

The efficiency of RED energy conversion with constant $R_{\mathrm{L}}$ ranges between 43.3 and $50.7 \%$ and only changes slightly with $\phi$ (solid blue line in Figure 3B). Hence, simplifying the RED stack design by restricting the external load to a constant resistance lowers the portion of $\Delta G_{\text {mix }}$ that can be converted to useful work by up to $\sim 7 \%$. Similar trends were observed for RED power generation with seawater-brackish water (Figure S5, Supporting Information), except with slightly better efficiency of between $47.1 \%$ and $52.2 \%$. As discussed earlier, the batch process analyzed here also depicts a continuous flow cocurrent RED stack. Because greater mixing can be achieved by employing counter- or cross-current flow configurations in a membrane stack, more energy per unit volume of the low concentration river water can be extracted. ${ }^{14}$

Losses Due to Stack Resistance. The difference between $W_{\text {ideal }}$ and useful work is attributed to power dissipated by the internal resistance of the RED salinity battery (i.e., $I^{2} r_{\text {stack }}$ ). During controlled mixing, ion fluxes in the RED cell are impeded by the resistance of the membranes and solution compartments. Entropy is produced when energy is expended to overcome these resistive forces. This dissipative loss (indicated in Figure $2 \mathrm{~B}$ as the red pattered region) is, thus, not available for utilization by the external load to perform useful work and is analogous to the irreversible energy loss due to entropy production in pressure retarded osmosis power generation and reverse osmosis desalination. ${ }^{4,36,37}$

When RED membrane power density is maximized, slightly over half of the salinity energy is lost through internal resistance dissipation. The portion of available energy lost to entropy production (red patterned region of Figure $2 \mathrm{~B}$ ) is larger at the beginning of the controlled mixing process (i.e., $0<\Delta n_{\mathrm{s}} / \Delta n_{\mathrm{s}}^{f}<$ $0.1)$ due to mismatch of the external load resistance to $r_{\text {stack. }}$. The energy efficiency, $\eta$, can be enhanced (e.g., $>50 \%$ ) by using an external load of higher resistance (eq 9). However, this improvement will be at the expense of a lower power density 
(eq 10), thus unfavorably raising the membrane area required. ${ }^{6,14}$ Detailed discussion on membrane power density performance and the relationship with $\eta$ is presented in a later section.

\section{INFLUENCE OF MEMBRANE SELECTIVITY ON ENERGY EFFICIENCY}

Thus far, the analyses have assumed ideal ion-exchange membranes with perfect selectivity. Specifically, the cation- and anion-exchange membranes completely reject co-ions and exclude water migration. In this section, we examine RED power generation for the more realistic case of imperfect membrane selectivity.

Selectivity Imperfections of Ion-Exchange Membranes. Ion-exchange membranes are water-swollen thin-film polymers that possess a high concentration of fixed charges (negative for CEMs and positive for AEMs) that allow the selective permeation of counterions by Donnan exclusion of the co-ions. $71,38-40$ Actual ion-exchange membranes exhibit imperfections in selectivity, specifically co-ion flux across the membranes, diffusion of water from low to high salt concentration solution due to the osmotic gradient, and electro-osmosis where water molecules are dragged along by the charged ions flux..$^{10,41,42}$ Here, we discuss these phenomena and evaluate the relative influence on RED energy efficiency. The cation- and anion-exchange membranes are assumed to have symmetrically identical selectivity. A detailed presentation on the effects can be found in the Supporting Information.

(a). Co-ion Transport. Because of Donnan exclusion, the concentration of co-ions within the charged membrane is much lower than counterions. ${ }^{7,38}$ The IEMs are, therefore, preferentially selective for counterions but still slightly permeable to coions. We introduce a dimensionless parameter, $\beta$, which quantifies the relative leakage of co-ions across the membranes

$$
\beta \equiv \frac{\Delta n_{\mathrm{s}, \mathrm{co}}}{\Delta n_{\mathrm{s}, \mathrm{ct}}}
$$

where $\Delta n_{\mathrm{s}}$ denotes the moles of salt transported across the membrane and subscripts "co" and "ct" indicate co-ions and counterions, respectively. A perfectly selective membrane that only allows counterion transport has $\beta$ of zero, whereas $\beta=1$ represents the transport of $\mathrm{Na}^{+}$and $\mathrm{Cl}^{-}$ions in equal pairs (i.e., nonselective membrane). Membrane permselectivity, $\alpha$, is related to $\beta$ by (detailed explanation is presented in the Supporting Information): ${ }^{43,44}$

$$
\alpha=\frac{1-\beta}{1+\beta}
$$

The relationship between $\beta$ and transport number, $t$ (defined as the fraction of total ions that is transported across the IEM as counter- or co-ions), is also presented in the Supporting Information.

(b). Osmosis. Ion-exchange membranes are hydrated polymeric thin films and, thus, are pervious to water. ${ }^{10,41}$ The salt concentration difference between the HC and LC solution produces an osmotic gradient across the IEMs and drives the osmosis of water. We define $D^{\mathrm{R}}$ as the ratio of water to salt (as coion) diffusivity

$$
D^{\mathrm{R}} \equiv \frac{D_{\mathrm{w}}}{D_{\mathrm{s}}}=-\frac{\Delta V_{\mathrm{os}}}{\Delta n_{\mathrm{s}, \mathrm{co}} \bar{V}}
$$

where $D_{\mathrm{w}}$ and $D_{\mathrm{s}}$ are the effective diffusion coefficients of water and salt transported as co-ions, respectively, in the membrane matrix. For the relatively dilute solutions considered in this study, the osmotic gradient can be approximated with the salt concentration difference. Assuming co-ion and water fluxes across the IEMs are governed by Fickian diffusion, ${ }^{45} D^{\mathrm{R}}$ is, thus, equal to the mole ratio of water permeated by osmosis $\left(\Delta V_{\text {os }} / \bar{V}\right.$, where $\Delta V_{\text {os }}$ is the volume and $\bar{V}$ is the molar volume of water) to salt transported as co-ions $\left(\Delta n_{\mathrm{s}, \mathrm{co}}\right)$. Detailed presentation of this relationship is shown in the Supporting Information. Note that the negative sign indicates water osmosis is in the opposite direction to salt flux. Osmosis is an uncontrolled mixing in RED and is, hence, deleterious to energy production.

(c). Electro-osmosis. Charged ions permeating across a hydrated IEM exert an electrostatic field that drags along nearby polar water molecules. ${ }^{41,42}$ This phenomenon, termed electroosmosis, induces a water flux in the direction of ion transport (i.e., against the osmotic gradient) and, thus, deducts from osmosis. Water migration due to electro-osmosis is proportional to the total ion flux ${ }^{10,42}$ by a dimensionless constant, $h$

$$
h=\frac{\Delta V_{\mathrm{eo}}}{\left(\Delta n_{\mathrm{s}, \mathrm{ct}}+\Delta n_{\mathrm{s}, \mathrm{co}}\right) \bar{V}}
$$

where $\Delta V_{\mathrm{eo}}$ is the volume of water transported by electroosmosis and $\Delta n_{\mathrm{s}, \mathrm{ct}}+\Delta n_{\mathrm{s}, \mathrm{co}}$ is the total moles of salt permeated. Unlike co-ion transport and osmosis, water migration caused by electro-osmosis is advantageous to RED power generation as it reduces the net water flux to the $\mathrm{HC}$ solution. In typical RED operation, the electro-osmotic flux is lower than the osmotic flux such that there is net water transport from the LC to the HC solution. $^{10}$

Uncontrolled Mixing Decreases Extractable Work. RED extracts work from the energy of mixing through the permeation of counterions across the Nernst potential of the ion-exchange membranes. Thus, selectivity imperfections in the membrane represent uncontrolled mixing that detrimentally lowers the power output. Derivation of the effect of uncontrolled mixing on extractable work is shown in the Supporting Information, and the key equations are briefly presented and discussed here.

Because of co-ion leakage and water osmosis, the fraction of salt in the initial HC solution that is transported as counterions is lowered

$$
\frac{\Delta n_{s}^{f}}{n_{s, \mathrm{HC}}^{0}}=\frac{\phi}{(1+\beta)\left(1+\theta \bar{V} c_{\mathrm{M}}^{f}\right)}\left(1-\frac{c_{\mathrm{LC}}^{0}}{c_{\mathrm{HC}}^{0}}\right)
$$

where $\theta$ is the mole ratio of water to salt (both counterions and co-ions) permeation across the IEMs. The mole ratio combines both effects of osmosis and electro-osmosis, and can be expressed as

$$
\theta \equiv \frac{\Delta V}{\left(\Delta n_{\mathrm{s}, \mathrm{ct}}+\Delta n_{\mathrm{s}, \mathrm{co}}\right) \bar{V}}=\frac{\beta}{1+\beta} D^{\mathrm{R}}-h
$$

where $\Delta V$ is the net volumetric water transport across the membranes (i.e., difference between osmotic flux and electroosmotic flux). Note that, henceforth, $\Delta n_{\mathrm{s}}$ denotes the moles of salt transported as counterions. Setting $\beta$ and $\theta$ to zero in eq 16 recovers eq 3 , the fraction of salt eventually transported for perfectly selective membranes.

The imperfect selectivity of the IEMs for counterions diminishes the effective electromotive force across the one-cell RED by the membrane permselectivity factor, $\alpha$ (eq 1 ). 
Additionally, the undesirable leakage of co-ions negates an equal charge of counterions permeating across the CEM and AEM (Figure 1B), thereby lowering the net ion flux. Factoring in these deleterious effects, an external load of infinitely large resistance was employed such that $\xi_{\mathrm{L}}$ approaches $\xi_{\text {emf }}$ and the maximum specific work (with infinitesimal ion flux and power density) was determined (detailed derivation is shown in the Supporting Information)

$$
\begin{aligned}
& W_{\text {Imp }, V_{\mathrm{LC}}^{0}}=z F \int_{0}^{\Delta n_{s}^{f}} \xi_{\text {emf }} \mathrm{d}(1-\beta) \Delta n_{s} \\
& =\alpha\left\{\Delta G_{\text {mix }, V_{\mathrm{LC}}^{0}}-\frac{\nu R_{g} T}{\theta \bar{V}}\left[\begin{array}{l}
\frac{1-\phi}{\phi}\left(1+\theta \bar{V} c_{\mathrm{HC}}^{0}\right) \ln \left(1+\theta \bar{V} c_{\mathrm{HC}}^{0}\right) \\
+\left(1+\theta \bar{V} c_{\mathrm{LC}}^{0}\right) \ln \left(1+\theta \bar{V} c_{\mathrm{LC}}^{0}\right) \\
-\frac{1}{\phi}\left(1+\theta \bar{V} c_{\mathrm{M}}^{f}\right) \ln \left(1+\theta \bar{V} c_{\mathrm{M}}^{f}\right)
\end{array}\right]\right\}
\end{aligned}
$$

where $W_{\text {Imp }, V^{0} \mathrm{LC}}$ is energy extractable in RED with imperfectly selective IEMs, per unit volume of the initial LC solution. Note that $\xi_{\text {emf }}$ is integrated from zero to $\Delta n_{\mathrm{s}}^{f}$ (eq 16) that has been lowered by co-ion leakage and water transport.

It is worthwhile to note that although RED proceeded with an infinitesimal ionic/electric current in this case, it is not a reversible thermodynamic process because entropy is produced in the uncontrolled mixing of co-ion transport and water osmosis between the $\mathrm{HC}$ and LC solution. As $\alpha \rightarrow 1$ (i.e., $\beta$ tends to zero) and $\theta \rightarrow 0$, the membranes approach perfect selectivity and the specific work (eq 18) reduces to the Gibbs free energy of mixing or $W_{\text {ideal, }, V_{\mathrm{LC}}^{0}}($ eq 6$)$. Conversely, if the ion-exchange membranes are completely unselective for counterions ( $\beta=1$ and, thus, $\alpha=$ $0)$, the net charge transfer is zero because the ions permeate across as cation-anion pairs and no work is produced.

The maximum specific work was analyzed in a seawater-river water RED salinity battery with IEM properties simulating actual commercial and laboratory-fabricated membranes. ${ }^{10,31,32}$ The permselectivity, $\alpha$, is 0.9 (i.e., $\beta=0.053)$ and $\theta=19\left(D^{\mathrm{R}}=500\right.$ and $h=6)$. Typical $\alpha$ values are $\sim 0.79-0.99,{ }^{10,31,32}$ while $D^{\mathrm{R}}$ and $h$ range between 100 and 725 and $4.6-12$, respectively. ${ }^{10}$ To illustrate the relative migration of species, when $1 \mathrm{~L}$ of $600 \mathrm{mM}$ $\mathrm{NaCl} \mathrm{HC}$ solution and $1 \mathrm{~L}$ of $1.5 \mathrm{mM} \mathrm{NaCl} \mathrm{LC}$ solution are employed in the one-cell RED depicted in Figure 1B, 260 mmol of salt permeates the membranes as counterions, $\sim 14$ mmol of $\mathrm{NaCl}$ diffuses across as co-ions, and $\sim 93 \mathrm{~mL}$ of water move from the LC solution to the $\mathrm{HC}$ solution (i.e., osmosis minus electro-osmosis) over the entire process.

Figure 4 shows $W_{\mathrm{Imp}, V_{\mathrm{LC}}^{0}}$ and the percentage of $\Delta G_{\text {mix }}$ lost in energy extraction, as a function of the volumetric fraction of the LC solution, $\phi$, for the IEMs described above (solid red lines). The individual effect of co-ion leakage and water osmosis (indicated by dash-dotted green lines and dotted blue lines, respectively) can be further separated out by respectively setting $\theta$ to zero or $\alpha=1$ in eq 18 . The ideal work (or, equivalently, $\left.\Delta G_{\text {mix }}\right)$ is plotted in Figure 4A for comparison. For the simulated imperfect selectivity membranes, co-ion leakage in RED caused a $19 \%$ decline in extractable energy, while net water permeation into the HC solution resulted in $\sim 2-9 \%$ decrease (Figure $4 \mathrm{~B}$ ). This result indicates that, for the currently available ion-exchange membranes, co-ion leakage has a greater impact on RED performance than water transport. The combined effects of selectivity imperfections detrimentally lowered the extractable energy by $\sim 21-26 \%$, with a greater loss experienced at smaller $\phi$ values. Co-ion and water osmosis is akin to uncontrolled mixing of the HC and LC solutions; thus, a portion of $\Delta G_{\text {mix }}$ is lost to

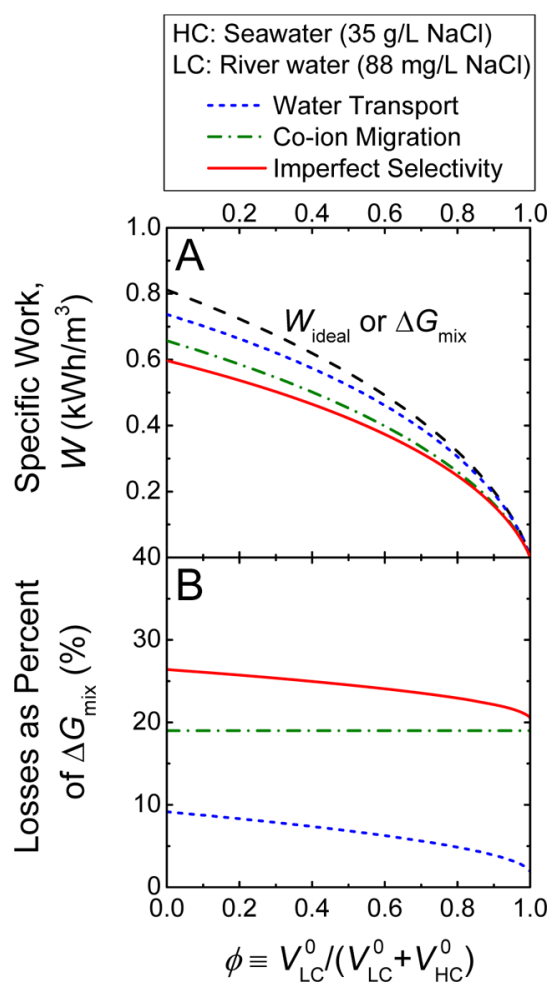

Figure 4. (A) Extractable work per unit volume of the LC solution, $W$, as a function of the volumetric fraction of LC solution to both LC and HC solutions, $\phi$, where the potential difference across the external load is always exactly equal to $\xi_{\text {emf }}$. The dashed black line indicates $W_{\text {ideal, } V_{\text {LC }}^{0}}$ or $\Delta G_{\text {mix }, V_{L C}^{0}}$ for perfectly selective ion-exchange membranes. The dotted blue line and dash-dotted green line denote $W$ for imperfect membrane selectivity where osmosis of water $(\theta=19)$ and transport of co-ions $(\alpha=$ $0.9)$ occur, respectively. The specific work for nonideal ion-exchange membranes having both selectivity imperfections (i.e., $\alpha=0.9$ and $\theta=$ 19) are indicate by the solid red line. (B) Percentage of Gibbs free energy of mixing (or ideal work) that is not extractable due to selectivity imperfections in the CEM and AEM, as a function of $\phi$. Lines represent the same imperfections as in (A). The temperature $T=298 \mathrm{~K}$, the HC solution is seawater $(600 \mathrm{mM}$ or $35 \mathrm{~g} / \mathrm{L} \mathrm{NaCl})$, and the $\mathrm{LC}$ solution is river water $(1.5 \mathrm{mM}$ or $88 \mathrm{mg} / \mathrm{L} \mathrm{NaCl})$.

entropy production and the process is rendered thermodynamically irreversible.

Imperfect Selectivity Reduces RED Power Generation Efficiency. Salinity battery performance when $\mathrm{PD}_{\text {avg }}$ is maximized was analyzed for two different extent of selectivity imperfection to simulate actual membranes: ion-exchange membranes B (designated IEM-B) were examined earlier and presented in Figure $4(\alpha=0.9$ and $\theta=19)$, while IEM-A represents more selective membranes with permselectivity $\alpha$ of 0.95 and $\theta$ of 3.5 (i.e., $\beta=0.026, D^{\mathrm{R}}=300$, and $h=4$ ).$^{10,31,32}$ The specific work, energy efficiency $\eta$, and overall power density as a function of $\phi$ are shown in Figure 3 for a seawater-river water system. Dashed magenta lines and dash-dotted red lines indicate membranes $\mathrm{A}$ and $\mathrm{B}$, respectively.

Leakage of co-ions and osmosis of water across the simulated CEMs and AEMs deleteriously reduce the fraction of Gibbs free energy of mixing that can be converted to useful work with RED. Perfectly selective membranes have $\eta$ of $43.3-50.7 \%$ (solid blue line), while the efficiencies of membranes $A$ and $B$ range between $38.7-45.6 \%$ and $32.7-40.4 \%$, respectively, with slightly higher $\eta$ achieved at larger $\phi$ values (Figure 3B). As expected, a lower 
selectivity (i.e., IEM-B) has a greater negative impact on RED energy production with natural salinity gradients. Therefore, imperfections in the selectivity of technologically available membranes cause $\sim 5-10 \%$ (absolute) of $\Delta G_{\text {mix }}$ to be lost to entropy production. Similar trends were observed when brackish water $(17 \mathrm{mM}$ or $1 \mathrm{~g} / \mathrm{L} \mathrm{NaCl})$ was used as the LC solution (Figure S5, Supporting Information).

\section{POWER DENSITY ANALYSIS}

Membrane power density, the power generated per unit total membrane area, is critical in determining the cost-effectiveness of RED energy production with natural salinity gradients. ${ }^{8,10,14,35}$ For instance, doubling $\mathrm{PD}_{\text {avg }}$ would halve the membrane area requirement in a salinity battery installation and, thus, lower the capital cost. In this section, we analyze the potential membrane power density of RED salinity batteries and examine the influencing factors.

Power Density of Perfect and Imperfect Membrane Selectivity. Figures 3C and S5C (Supporting Information) show $\mathrm{PD}_{\mathrm{avg}}$ for a seawater $\mathrm{HC}$ solution paired with river water and brackish water, respectively, as a function of the volumetric fraction of the LC solution, $\phi$. Solid blue lines indicate perfectly selective CEM and AEM, while imperfect selectivities IEM-A and IEM-B are represented by dashed magenta lines and dash-dotted red lines, respectively. Note that although only one RED cell is considered in this analysis, power density is normalized by the membrane area, and hence, the $\mathrm{PD}_{\text {avg }}$ values obtained are representative of a module-scale RED salinity battery with multiple membrane-pair stacks.

For the seawater-river water system with IEM-A (dashed magenta line in Figure $3 \mathrm{C}), \mathrm{PD}_{\text {avg }}$ is lowest $\left(0.04 \mathrm{~W} / \mathrm{m}^{2}\right)$ when $\phi$ $\rightarrow 0$ and slowly increases with $\phi$ before rapidly rising to a peak of $0.65 \mathrm{~W} / \mathrm{m}^{2}$ when $\phi$ is $\sim 0.99$. The overall membrane power density is below $0.50 \mathrm{~W} / \mathrm{m}^{2}$ over a wide range of $\phi$ values (from 0 to 0.94$)$. As expected, $\mathrm{PD}_{\mathrm{avg}}$ of the perfectly selective membranes is slightly larger (solid blue line). Less selective IEM-B has lower $\mathrm{PD}_{\text {avg }}$ compared to IEM-A. The power density achievable with perfectly selective membranes and a variable resistance external load, denoted by the dotted green line, signifies the maximum $\mathrm{PD}_{\text {avg }}$ that can be attained. The power density of this hypothetical scenario is slightly higher than the three actual scenarios with constant $R_{\mathrm{L}}$ but otherwise displayed similar trends. When brackish water was employed as the LC solution (Figure S5C, Supporting Information), PD $_{\text {avg }}$ exhibited similar behavior over $\phi$, albeit at slightly lower magnitude.

Relatively higher membrane power density can be achieved by operating RED at very large $\phi$ values (i.e., pairing a large LC solution volume with a small amount of $\mathrm{HC}$ solution). For instance, in the seawater-river water system (Figure 3C), peak $\mathrm{PD}_{\text {avg }}$ of 0.65 and $0.54 \mathrm{~W} / \mathrm{m}^{2}$ is attained for IEM-A and IEM-B, respectively, when $\phi$ is $\sim 0.99$ (i.e., pairing one volume of seawater HC solution with 99 volumes of river water LC solution). The comparatively greater power density is attributed to a larger $\xi_{\text {emf }}$ being maintained over the RED duration (as illustrated in Figure S1B, Supporting Information). However, when $\phi=0.99$, the amount of useful work produced per unit LC solution volume, specific $W^{*}$, is 0.014 and $0.012 \mathrm{kWh} / \mathrm{m}^{3}$ with ion-exchange membranes $A$ and $B$, respectively, relatively minute compared to the specific $W^{*}$ attainable at smaller $\phi$ values (Figures 3A). In power generation from natural salinity gradients, often seawater from the ocean is abundant while fresh water from estuaries is the limiting resource. As such, obtaining the most out of the available energy from the finite river water input is anticipated to be a vital performance goal. Therefore, operating RED at very large $\phi$ is unlikely to be feasible despite the potential for higher power density. Hereafter, the analysis will focus on performance with $\phi=0.5$, i.e., equal volumes of $\mathrm{HC}$ and $\mathrm{LC}$ solution.

Enhancing Power Density by Energy Efficiency Tradeoff. An inspection of eqs 1 and 8 reveals that the ion flux across the IEMs rapidly diminishes toward the end of RED (i.e., $\Delta n^{\mathrm{s}} \rightarrow$ $\left.\Delta n_{\mathrm{s}}^{f}\right)$. The retarding rate of ion transport unfavorably draws out the process duration and detrimentally reduces $\mathrm{PD}_{\text {avg }}$ (eq 11). Recall that, to avoid obtaining a trivial solution when numerically determining $\mathrm{PD}_{\text {avg }}$ 99.99\% $\Delta n_{\mathrm{s}}^{f}$ transfer was taken as the process conclusion. Figure S6 of Supporting Information shows the potential difference across the external load and instantaneous $\mathrm{PD}$ as a function of fraction of salt permeated and normalized time-area, for a seawater-river water system with $\phi=0.5$. The protraction effect is especially evident in Figure S6D (Supporting Information), where PD initially rises and then precipitously dwindles to a minutely low, but nonzero, level for an extended period (inset of Figure S6D, Supporting Information). Useful work is still being produced by the external load, but the amount is negligibly small (area under $\xi_{\mathrm{L}}-\Delta n_{\mathrm{s}}$ plot in Figure S6A, Supporting Information). Therefore, energy extraction efficiency and power density of RED fades drastically as the process approaches its finish.

Overall membrane power density performance can, hence, be improved by judiciously discontinuing the process earlier when RED is no longer adequately effective. The remaining salinity energy embedded in the unmixed HC and LC solutions is, thus, not converted to useful work by the external load. The trade-off can be overall advantageous if the benefit from $\mathrm{PD}_{\mathrm{avg}}$ enhancement outweighs the sacrifice in work extraction. Figure 5A shows $\mathrm{PD}_{\text {avg }}$ (red patterned columns, left vertical axis) and $\eta$ (blue symbols, right vertical axis) after 99.99, 90, 80, 70, 60, and $50 \%$ of $\Delta n_{\mathrm{s}}^{f}$ has permeated across the membranes for $\phi=0.5$. Model seawater and river water were employed as the $\mathrm{HC}$ and LC solutions, respectively, and moderately selective IEM-A ( $\alpha=$ 0.95 and $\theta=3.5$ ) was used. Power density and efficiency over the entire range of $\phi$ for $\Delta n_{\mathrm{s}}^{f}$ permeation of $30-99.99 \%$ is presented in Figure S7 of the Supporting Information.

When $90 \%$ and $80 \%$ of $\Delta n_{\mathrm{s}}^{\dagger}$ has permeated (or, alternatively, $90 \%$ and $80 \%$ of the charges are utilized), $\eta$ is $39.6 \%$ and $39.0 \%$, respectively, similar to the efficiency of $39.1 \%$ with $99.99 \% \Delta n_{\mathrm{s}}^{f}$ permeation (Figure 5A). On the other hand, overall power density increases dramatically by 4.6 and 6.5 times to 0.42 and $0.59 \mathrm{~W} / \mathrm{m}^{2}$, respectively (compared with power density of 0.09 $\mathrm{W} / \mathrm{m}^{2}$ for $99.99 \%$ charge utilization). Hence, by forgoing the last $10-20 \%$ of $\Delta n_{\mathrm{s}}, \mathrm{PD}_{\text {avg }}$ performance is substantially enhanced without significantly affecting $\eta$. When the process is ceased even earlier (i.e., 70, 60, and 50\% charge utilization), $\eta$ is $37.8,35.8$, and $33.2 \%$ and $\mathrm{PD}_{\text {avg }}$ is $0.77,0.96$, and $1.16 \mathrm{~W} / \mathrm{m}^{2}$, respectively, for $\phi=0.5$ (Figure 5A). Although power density is considerably improved, the corresponding reduction in efficiency becomes noticeably substantial. Further reducing the charge utilization to $40 \%$ and $30 \%$ yields even higher power density (Figure S7, Supporting Information). However, the energy efficiency is concomitantly lowered to diminished levels that could potentially render the RED power generation process unviable. Similar trends are observed, albeit with slightly lower power densities, when brackish water is employed as the LC solution and for less selective IEM-B (results not shown).

Influence of Intermembrane Distance and Membrane

Resistance on Power Density. The above analysis indicates 

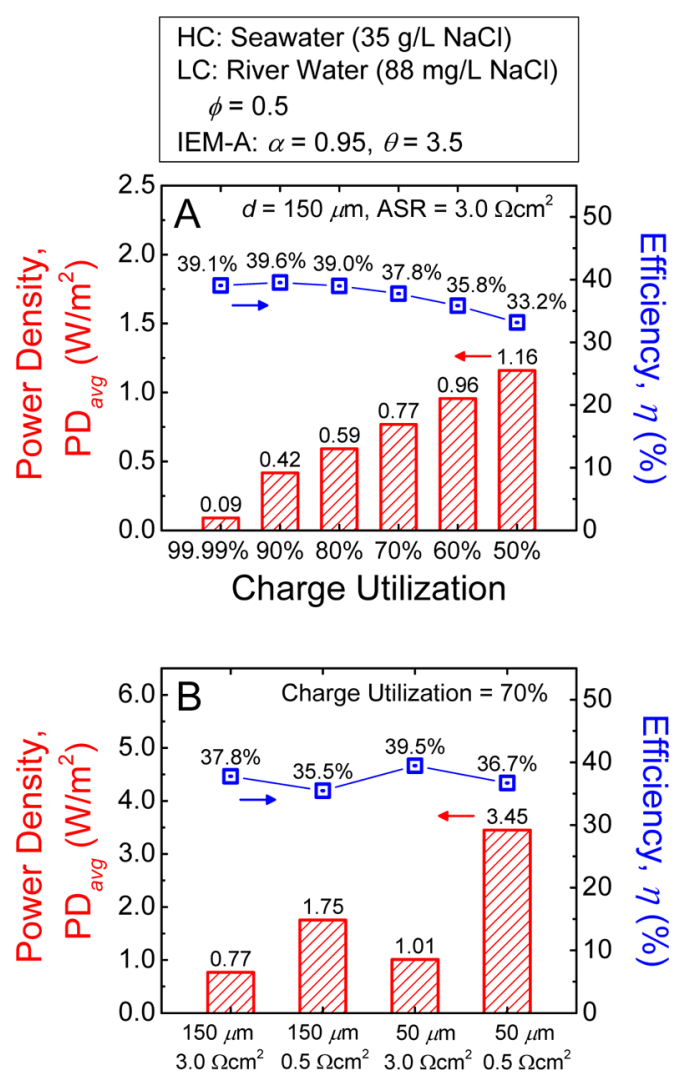

Figure 5. Overall membrane power density, $\mathrm{PD}_{\text {avg }}$ (left vertical axis), and energy conversion efficiency, $\eta$ (right vertical axis), for seawaterriver water RED power generation with $\phi=0.5$. (A) The controlled mixing process is terminated prematurely, thereby utilizing only the earlier transported charges for power generation and discarding the remainder. A range of charge utilization percentages (99.99, 90, 80, 70, 60 , and $50 \%)$ are evaluated. For this analysis, the intermembrane distance, $d$, is $150 \mu \mathrm{m}$ and the ion-exchange membrane area specific resistance, ASR, is $3.0 \Omega \mathrm{cm}^{2}$. (B) Different intermembrane distances ( $d$ $=50$ or $150 \mu \mathrm{m}$ ) and membrane area specific resistances (ASR $=0.5$ or $3.0 \Omega \mathrm{cm}^{2}$ ) are employed to examine the effect on RED performance. The charge utilization is $70 \%$ for the four scenarios. In both plots, the ion-exchange membranes have moderately imperfect selectivity (i.e., IEM-A with $\alpha=0.95$ and $\theta=3.5)$. Equal volumes of seawater $(600 \mathrm{mM}$ or $35 \mathrm{~g} / \mathrm{L} \mathrm{NaCl})$ and river water $(1.5 \mathrm{mM}$ or $88 \mathrm{mg} / \mathrm{L} \mathrm{NaCl}$ ) were used (i.e., $\phi=0.5$ ), and temperature $T=298 \mathrm{~K}$.

that, with IEM area specific resistance of $3 \Omega \mathrm{cm}^{2}$ and intermembrane distance of $150 \mu \mathrm{m}$, the low overall membrane power density will pose a significant barrier to the cost-effective implementation of RED power generation with natural salinity gradients. An inspection of eq 10 indicates that reducing the internal resistance of the repeating $\mathrm{RED}$ cells can improve $\mathrm{PD}_{\text {avg. }}$. Specifically, the CEMs and AEMs need to be more conductive and the intermembrane spacing reduced (eq 7). Efficiency, $\eta$, and $\mathrm{PD}_{\text {avg }}$ were analyzed for an additional three hypothetical scenarios: (i) the area specific resistance of the IEMs is lowered to $0.5 \Omega \mathrm{cm}^{2}$, (ii) the intermembrane distance, $d$, is reduced to 50 $\mu \mathrm{m}$, and (iii) combining both ASR $=0.5 \Omega \mathrm{cm}^{2}$ and $d=50 \mu \mathrm{m}$. The results are compared to the previous analysis where membrane ASR $=3.0 \Omega \mathrm{cm}^{2}$ and $d=150 \mu \mathrm{m}$ and presented in Figure 5B. Moderate selectivity ion-exchange membranes were employed (i.e., IEM-A with $\alpha=0.95$ and $\theta=3.5$ ), and the process was discontinued after $70 \%$ of $\Delta n_{\mathrm{s}}^{f}$ was permeated. Seawater was used as the $\mathrm{HC}$ solution, the $\mathrm{LC}$ solution is river water, and $\phi=$ 0.5 . Figuress S8A and S8B (Supporting Information) show $\eta$ and
$\mathrm{PD}_{\text {avg }}$, respectively, for $70 \%$ and $50 \%$ charge utilization over the entire range of $\phi$.

To simulate highly conductive CEMs and AEMs, an areaspecific resistance of $0.5 \Omega \mathrm{cm}^{2}$ was selected (approximately the lowest reported ASR by membrane manufacturers). ${ }^{31}$ When membrane resistance is decreased from $3.0 \Omega \mathrm{cm}^{2}$ to $0.5 \Omega \mathrm{cm}^{2}$, power density was substantially enhanced 2.3 -fold, from 0.77 to $1.75 \mathrm{~W} / \mathrm{m}^{2}$ (red patterned columns in Figure 5B), underscoring the importance of membrane conductivity in PD performance. As illustrated in Figures S3 (Supporting Information), the IEMs dominate the RED cell resistance for most part of the process. Employing more conductive membranes, thus, significantly reduces $\mathrm{ASR}_{\text {cell }}$ and enables faster ionic transport to produce greater power densities. Comparing $\eta$ (blue symbols in Figure $5 \mathrm{~B})$, the more conductive membranes are slightly less efficient in extracting salinity energy ( $\eta$ is $35.5 \%$ and $37.8 \%$ when membrane ASR is 0.5 and $3.0 \Omega \mathrm{cm}^{2}$, respectively).

The resistance of the solution compartments is directly proportional to the intermembrane distance $($ ASR $=d / \kappa)$. Therefore, reducing the CEM-AEM spacing from 150 to $50 \mu \mathrm{m}$ (approximately the lowest experimentally investigated distance $)^{13}$ trims the area specific resistance of the LC compartment, a major contributor to RED stack resistance, down by twothirds. Compared to the case when $d=150 \mu \mathrm{m}$, the reduced intermembrane distance moderately enhances $\mathrm{PD}_{\text {avg }}$ by 1.3 times of $0.77 \mathrm{~W} / \mathrm{m}^{2}$ (red patterned columns in Figure $5 B$ ). The narrower solution compartment width suppresses $\mathrm{ASR}_{\text {cell }}$ and facilitates faster ion permeation across the RED cell, resulting in higher power density performance. Recall that the LC solution dominates $\mathrm{ASR}_{\text {cell }}$ initially (Figure S3, Supporting Information) and cause dissipative losses to be most pronounced at the start of the controlled mixing (Figure 2B). The lower $d$ allowed better matching of constant load resistance to the early $\mathrm{ASR}_{\text {cell }}$ and, thereby, increase the energy extraction efficiency (blue symbols). At $\phi=0.5$ in a seawater-river water system with $70 \%$ charge utilization, $\mathrm{PD}_{\text {avg }}$ is $1.01 \mathrm{~W} / \mathrm{m}^{2}$ and $\eta$ is $39.5 \%$.

Simultaneously decreasing IEM area specific resistance and intermembrane distance (to $0.5 \Omega \mathrm{cm}^{2}$ and $50 \mu \mathrm{m}$, respectively) enhances power density considerably by 4.5 times (red patterned columns in Figure 5B). Compared to the initial analysis (i.e., 3.0 $\Omega \mathrm{cm}^{2}$ and $\left.150 \mu \mathrm{m}\right)$, the energy conversion efficiency is marginally lower (blue symbols). At $\phi=0.5$ with $70 \%$ charge utilization, $\mathrm{PD}_{\text {avg }}=3.45 \mathrm{~W} / \mathrm{m}^{2}$ and $\eta=36.7 \%$ with the smaller membrane ASR and $d$. On the other hand, with membrane ASR of $3.0 \Omega \mathrm{cm}^{2}$ and $d=150 \mu \mathrm{m}$, the power density and efficiency are $0.77 \mathrm{~W} / \mathrm{m}^{2}$ and $\eta=37.8 \%$, respectively. Note that the power density benefit acquired from the simultaneous enhancement in membrane conductivity and reduction in channel thickness is greater than the cumulative sum of each individual improvement $(4.5>2.3 \times 1.3)$. This suggests that greater gains can be obtained by concurrently targeting the membrane and module design. Figures S8C and S8D (Supporting Information) show performance for $0<\phi<1$ when the same RED system is discontinued after $50 \%$ of $\Delta n_{\mathrm{s}}^{f}$ has permeated across the membranes. Power densities up to $5.95 \mathrm{~W} / \mathrm{m}^{2}$ can be achieved, but at the expense of lower work extraction efficiencies. For instance, when membrane ASR $=0.5 \Omega \mathrm{cm}^{2}$ and the intermembrane distance is $50 \mu \mathrm{m}$, $\mathrm{PD}_{\text {avg }}$ and $\eta$ attainable are $4.99 \mathrm{~W} / \mathrm{m}^{2}$ and $32.5 \%$, respectively, for $\phi=0.5$. 


\section{IMPLICATIONS FOR NATURAL SALINITY GRADIENT POWER GENERATION}

Energy efficiency and membrane power density will both be critical performance objectives that affect the feasibility of RED power generation with natural salinity gradients. The analysis presented in this study shows an upper bound on both the highest actual efficiency attainable and the corresponding membrane power density achievable. Additionally, the two performance parameters are inextricably linked: enhancing the power density will inevitably necessitate a sacrifice in efficiency and vice versa. Furthermore, obtaining the most energy per unit volume of fresh water, likely to be the limiting resource, will also be a key performance target. This, together with the practical need for pretreatment of the feed streams to mitigate membrane fouling, ${ }^{46,47}$ is anticipated to constrain the fresh water-seawater mixing proportions that can feasibly be implemented. Actual RED power generation will need to work within such practical limitation and, at the same time, balance the intricate relationships between specific work, energy conversion efficiency, and power density when fine-tuning the operating parameters to optimize the overall cost-effectiveness of the technology.

At the heart of the RED salinity battery are ion-exchange membranes. Improving permselectivity and minimizing water osmosis across the membranes can curb undesired entropy production from uncontrolled mixing. The analysis here indicates that co-ion transport has a greater detrimental impact on RED power generation performance than water leakage. Feasibility studies indicate that advancement of the technology hinges on attaining higher power densities than currently available membranes. ${ }^{35,48}$ Our study quantitatively demonstrates that there is huge potential for power density enhancement by developing more conductive membranes. However, recent investigations also suggest that a trade-off relationship between ionic conductance and permselectivity exists for ion exchange membranes, where higher permselectivity is inevitably accompanied by lower membrane conductance. ${ }^{31,49}$ Likewise, reducing membrane resistance by fabricating thinner membranes is accompanied by greater co-ion transport and water permeation. $^{32}$ Employing novel materials or casting techniques in membrane fabrication can potentially advance CEMs and AEMs beyond the existing trade-off relationship and improve RED performance. However, given membranes is a major component of the initial capital outlay and the current high cost of membranes, ${ }^{48}$ there is most room for improving the costeffectiveness of the technology by developing more affordable ion-exchange membranes while retaining performance.

Reducing the intermembrane distance of the RED cells lowers the stack internal resistance but poses significant engineering challenges from the concomitant increase in the parasitic pressure drop along the channel. ${ }^{18}$ More energy is needed to push fluids through the narrower channel, and, hence, the pumping cost is detrimentally raised. For a laminar flow in a rectangular channel, the pressure drop is inversely proportional to the cube of the channel height. ${ }^{50}$ Thus, lowering the intermembrane spacing by a third, e.g., from 150 to $50 \mu \mathrm{m}$, will increase the head loss by a massive 27 times. Additionally, to maintain such a tiny gap between the CEMs and AEMs, channel spacers will be necessary. ${ }^{13}$ However, such spacers further hinder flow and exacerbate the pressure drop along the channel. A previous study indicates that operating RED at a small intermembrane distance of $60 \mu \mathrm{m}$ with spacers will negatively impact power generation because the large pumping energy consumed overwhelms the power density benefits. ${ }^{13}$ Inventive solutions, such as profiled membranes, ion conductive spacers, and innovative channel design, ${ }^{20,51,52}$ are needed to overcome the resistance and hydrodynamic constraints, in order to realize RED power generation with natural salinity gradients.

\section{ASSOCIATED CONTENT}

\section{Supporting Information}

Detailed presentation of the potential across a reverse electrodialysis cell; approximation of mole fraction to volumetric fraction; contributions to the internal resistance of one RED cell; detailed presentation on the effect of ion exchange membrane selectivity imperfections-co-ion transport, osmosis, and electro-osmosis-on RED energy production; nomenclature; electric potential across one RED cell as a function of $\Delta n_{\mathrm{s}} / n_{\mathrm{s}, \mathrm{HC}}^{0}$ and $\Delta n_{\mathrm{s}} / \Delta n_{\mathrm{s}}^{f}$ (Figure S1); specific ideal work, for river water and brackish water paired with seawater, as a function of $\phi$ (Figure S2); representative plot of the area specific resistance of the RED cell as a function of $\Delta n_{\mathrm{s}} / \Delta n_{\mathrm{s}}^{f}$ (Figure S3); schematic of an RED circuit (Figure S4); extractable work, energy efficiency, and power density as a function of $\phi$ for brackish water-seawater (Figure S5); potential difference across the external load resistor and power density as a function of process progression (Figure S6); energy efficiency and power density as a function of $\phi$ for a range of charge utilization (Figure S7); energy efficiency and power density as a function of $\phi$ for different membrane resistances and intermembrane distances (Figure S8); This material is available free of charge via the Internet at http://pubs. acs.org.

\section{AUTHOR INFORMATION}

\section{Corresponding Author}

*Phone: +1 203432 2789. E-mail: menachem.elimelech@yale. edu.

\section{Notes}

The authors declare no competing financial interest.

\section{ACKNOWLEDGMENTS}

We acknowledge the Graduate Fellowship (to Ngai Yin Yip) made by the Environment and Water Industrial Development Council of Singapore.

\section{REFERENCES}

(1) Chu, S.; Majumdar, A. Opportunities and challenges for a sustainable energy future. Nature 2012, 488 (7411), 294-303.

(2) Pattle, R. E. Production of electric power by mixing fresh and salt water in the hydroelectric pile. Nature 1954, 174 (4431), 660-660.

(3) Logan, B. E.; Elimelech, M. Membrane-based processes for sustainable power generation using water. Nature 2012, 488 (7411), 313-319.

(4) Yip, N. Y.; Elimelech, M. Thermodynamic and energy efficiency analysis of power generation from natural salinity gradients by pressure retarded osmosis. Environ. Sci. Technol. 2012, 46 (9), 5230-5239.

(5) Weinstein, J. N.; Leitz, F. B. Electric-power from difference in salinity - dialytic battery. Science 1976, 191 (4227), 557-559.

(6) Post, J. W.; Hamelers, H. V. M; Buisman, C. J. N. Energy recovery from controlled mixing salt and fresh water with a reverse electrodialysis system. Environ. Sci. Technol. 2008, 42 (15), 5785-5790.

(7) Baker, R. W. Membrane Technology and Applications, 3rd ed.; John Wiley \& Sons: Chichester, 2012; p xiv, 575 pp.

(8) Dlugolecki, P.; Gambier, A.; Nijmeijer, K.; Wessling, M. Practical potential of reverse electrodialysis as process for sustainable energy generation. Environ. Sci. Technol. 2009, 43 (17), 6888-6894. 
(9) Turek, M.; Bandura, B. Renewable energy by reverse electrodialysis. Desalination 2007, 205 (1-3), 67-74.

(10) Veerman, J.; de Jong, R. M.; Saakes, M.; Metz, S. J.; Harmsen, G. J. Reverse electrodialysis: Comparison of six commercial membrane pairs on the thermodynamic efficiency and power density. J. Membr. Sci. 2009, $343(1-2), 7-15$.

(11) Veerman, J.; Saakes, M.; Metz, S. J.; Harmsen, G. J. Reverse electrodialysis: Performance of a stack with 50 cells on the mixing of sea and river water. J. Membr. Sci. 2009, 327 (1-2), 136-144.

(12) Veerman, J.; Saakes, M.; Metz, S. J.; Harmsen, G. J. Electrical power from sea and river water by reverse electrodialysis: a first step from the laboratory to a real power plant. Environ. Sci. Technol. 2010, 44 (23), 9207-9212.

(13) Vermaas, D. A.; Saakes, M.; Nijmeijer, K. Doubled power density from salinity gradients at reduced intermembrane distance. Environ. Sci. Technol. 2011, 45 (16), 7089-7095.

(14) Vermaas, D. A.; Veerman, J.; Yip, N. Y.; Elimelech, M.; Saakes, M.; Nijmeijer, K. High efficiency in energy generation from salinity gradients with reverse electrodialysis. ACS Sustainable Chem. Eng. 2013, 1 (10), $1295-1302$

(15) Feinberg, B. J.; Ramon, G. Z.; Hoek, E. M. V. Thermodynamic analysis of osmotic energy recovery at a reverse osmosis desalination plant. Environ. Sci. Technol. 2013, 47 (6), 2982-2989.

(16) Daniilidis, A.; Vermaas, D. A.; Herber, R.; Nijmeijer, K. Experimentally obtainable energy from mixing river water, seawater or brines with reverse electrodialysis. Renew Energ 2014, 64, 123-131.

(17) Brauns, E. Salinity gradient power by reverse electrodialysis: effect of model parameters on electrical power output. Desalination 2009, 237 (1-3), 378-391.

(18) Vermaas, D. A.; Guler, E.; Saakes, M.; Nijmeijer, K. Theoretical power density from salinity gradients using reverse electrodialysis. Technoport 2012 - Sharing Possibilities and 2nd Renewable Energy Res. Conf. (RERC 2012) 2012, 20, 170-184.

(19) Tedesco, M.; Cipollina, A.; Tamburini, A.; van Baak, W.; Micale, G. Modelling the reverse electrodialysis process with seawater and concentrated brines. Desalin Water Treat 2012, 49 (1-3), 404-424.

(20) Veerman, J.; Saakes, M.; Metz, S. J.; Harmsen, G. J. Reverse electrodialysis: A validated process model for design and optimization. Chem. Eng. J. 2011, 166 (1), 256-268.

(21) Veerman, J.; Post, J. W.; Saakes, M.; Metz, S. J.; Harmsen, G. J. Reducing power losses caused by ionic shortcut currents in reverse electrodialysis stacks by a validated model. J. Membr. Sci. 2008, 310 (12), 418-430.

(22) Veerman, J.; Saakes, M.; Metz, S. J.; Harmsen, G. J. Reverse electrodialysis: evaluation of suitable electrode systems. J. Appl. Electrochem. 2010, 40 (8), 1461-1474.

(23) Burheim, O. S.; Seland, F.; Pharoah, J. G.; Kjelstrup, S. Improved electrode systems for reverse electro-dialysis and electro-dialysis. Desalination 2012, 285, 147-152.

(24) Scialdone, O.; Guarisco, C.; Grispo, S.; Angelo, A. D.; Galia, A. Investigation of electrode material - Redox couple systems for reverse electrodialysis processes. Part I: Iron redox couples. J. Electroanal. Chem. 2012, 681, 66-75.

(25) Vermaas, D. A.; Veerman, J.; Saakes, M.; Nijmeijer, K. Influence of multivalent ions on renewable energy generation in reverse electrodialysis. Energ Environ. Sci. 2014, 7 (4), 1434-1445.

(26) Smith, J. M.; Van Ness, H. C.; Abbott, M. M. Introduction to Chemical Engineering Thermodynamics, 7th ed.; McGraw-Hill: Boston, 2005; p xviii, 817 pp.

(27) Sandler, S. I. Chemical and Engineering Thermodynamics, 3rd ed.; Wiley: New York, 1999; p xx, 772 p.

(28) Długołęcki, P.; Anet, B.; Metz, S. J.; Nijmeijer, K.; Wessling, M. Transport limitations in ion exchange membranes at low salt concentrations. J. Membr. Sci. 2010, 346 (1), 163-171.

(29) Robinson, R. A.; Stokes, R. H. Electrolyte Solutions; The Measurement and Interpretation of Conductance, Chemical Potential, and Diffusion in Solutions of Simple Electrolytes, 2nd ed.; Butterworths: London, 1959; p xv, 571 p.
(30) Vermaas, D. A.; Saakes, M.; Nijmeijer, K. Enhanced mixing in the diffusive boundary layer for energy generation in reverse electrodialysis. J. Membr. Sci. 2014, 453, 312-319.

(31) Dlugolecki, P.; Nymeijer, K.; Metz, S.; Wessling, M. Current status of ion exchange membranes for power generation from salinity gradients. J. Membr. Sci. 2008, 319 (1-2), 214-222.

(32) Güler, E.; Elizen, R.; Vermaas, D. A.; Saakes, M.; Nijmeijer, K. Performance-determining membrane properties in reverse electrodialysis. J. Membr. Sci. 2013, 446, 266-276.

(33) Lee, K. L.; Baker, R. W.; Lonsdale, H. K. Membranes for powergeneration by pressure-retarded osmosis. J. Membr. Sci. 1981, 8 (2), 141-171.

(34) Yip, N. Y.; Tiraferri, A.; Phillip, W. A.; Schiffrnan, J. D.; Hoover, L. A.; Kim, Y. C.; Elimelech, M. Thin-film composite pressure retarded osmosis membranes for sustainable power generation from salinity gradients. Environ. Sci. Technol. 2011, 45 (10), 4360-4369.

(35) Post, J. W.; Goeting, C. H.; Valk, J.; Goinga, S.; Veerman, J.; Hamelers, H. V. M; Hack, P. J. F. M. Towards implementation of reverse electrodialysis for power generation from salinity gradients. Desalin Water Treat 2010, 16 (1-3), 182-193.

(36) Elimelech, M.; Phillip, W. A. The future of seawater desalination: energy, technology, and the environment. Science 2011, 333 (6043), $712-717$.

(37) Liu, C.; Rainwater, K.; Song, L. F. Energy analysis and efficiency assessment of reverse osmosis desalination process. Desalination 2011, $276(1-3), 352-358$.

(38) Mulder, M. Basic Principles of Membrane Technology, 2nd ed.; Kluwer Academic: Dordrecht, 1996; 564 pp.

(39) Geise, G. M.; Falcon, L. P.; Freeman, B. D.; Paul, D. R. Sodium chloride sorption in sulfonated polymers for membrane applications. $J$. Membr. Sci. 2012, 423-424, 195-208.

(40) Galama, A. H.; Post, J. W.; Cohen Stuart, M. A.; Biesheuvel, P. M. Validity of the Boltzmann equation to describe Donnan equilibrium at the membrane-solution interface. J. Membr. Sci. 2013, 442, 131-139.

(41) Belfort, G. Synthetic Membrane Processes: Fundamentals and Water Applications; Academic Press: Orlando, 1984; p xiii, 552 pp.

(42) Spiegler, K. S. Transport processes in ionic membranes. Trans. Faraday Soc. 1958, 54, 1408-1428.

(43) Lakshminarayanaiah, N. Transport phenomena in artificial membranes. Chem. Rev. 1965, 65 (5), 491-565.

(44) Strathmann, H. Ion-Exchange Membrane Separation Processes, 1st ed.; Elsevier: Amsterdam, 2004; p xi, 348 pp.

(45) Mackie, J. S.; Meares, P. The diffusion of electrolytes in a cationexchange resin membrane. I. Theoretical. Proc. R. Soc. London Ser. A 1955, 232 (1191), 498-509.

(46) Vermaas, D. A.; Kunteng, D.; Saakes, M.; Nijmeijer, K. Fouling in reverse electrodialysis under natural conditions. Water Res. 2013, 47 (3), $1289-1298$

(47) Vermaas, D. A.; Kunteng, D.; Veerman, J.; Saakes, M.; Nijmeijer, K. Periodic feedwater reversal and air sparging as antifouling strategies in reverse electrodialysis. Environ. Sci. Technol. 2014, 48 (5), 3065-3073.

(48) Daniilidis, A.; Herber, R; Vermaas, D. A. Upscale potential and financial feasibility of a reverse electrodialysis power plant. Appl. Energy 2014, 119, 257-265.

(49) Geise, G. M.; Hickner, M. A.; Logan, B. E. Ionic resistance and permselectivity tradeoffs in anion exchange membranes. ACS Appl. Mater. Interfaces 2013, 5, 10294-10301.

(50) Bird, R. B.; Stewart, W. E.; Lightfoot, E. N. Transport phenomena, 2nd ed.; Wiley: New York, 2002; p xii, 895 p.

(51) Vermaas, D. A.; Saakes, M.; Nijmeijer, K. Power generation using profiled membranes in reverse electrodialysis. J. Membr. Sci. 2011, 385 $(1-2), 234-242$.

(52) Długołęcki, P.; Dąbrowska, J.; Nijmeijer, K.; Wessling, M. Ion conductive spacers for increased power generation in reverse electrodialysis. J. Membr. Sci. 2010, 347 (1-2), 101-107. 